\title{
Nonstationary Filtering for Markov Jumping Systems with Fading Channel and Multiplicative Noises
}

\author{
Yang Zhan, Daijun Wei* \\ School of Science, Hubei University for Nationalities, Enshi, China \\ Email: *prof.wei@hotmail.com
}

How to cite this paper: Zhan, Y. and Wei, D.J. (2021) Nonstationary Filtering for Markov Jumping Systems with Fading Channel and Multiplicative Noises. Journal of Applied Mathematics and Physics, 9, 529-544.

https://doi.org/10.4236/jamp.2021.94037

Received: March 5, 2021

Accepted: March 30, 2021

Published: April 2, 2021

Copyright (c) 2021 by author(s) and Scientific Research Publishing Inc. This work is licensed under the Creative Commons Attribution International License (CC BY 4.0).

http://creativecommons.org/licenses/by/4.0/

\begin{abstract}
This paper studies the nonstationary filtering problem of Markov jump system under $l_{2}-l_{\infty}$ performance. Due to the difference in propagation channels, signal strength and phase will inevitably change randomly and cause the waste of signals resources. In response to this problem, a channel fading model with multiplicative noise is introduced. And then a nonstationary filter, which receives signals more efficiently is designed. Meanwhile Lyapunov function is constructed for error analysis. Finally, the gain matrix for filtering is obtained by solving the matrix inequality, and the results showed that the nonstationary filter converges to the stable point more quickly than the traditional asynchronous filter, the stability of the designed filter is verified.
\end{abstract}

\section{Keywords}

Markov Jumping System, $l_{2}-l_{\infty}$ Performance, Fading Channel,

Nonstationary Filtering, Multiplicative Noises

\section{Introduction}

Since entering the 21st century, the control process is increasingly complex, the control target is more diverse, the control precision requirements are more accurate, the system environment is more strict, the productivity of the world in the rapid development at the same time also faced with a variety of new challenges. For example, a large control system is usually formed by multiple subsystems acting together through time series and following one or more constraints. However, Markov jump system can well describe the control process in which multiple subsystems have random changes in a discrete time in the control process, and meet the needs of current practical application engineering. 
Due to its powerful modeling ability, Markov jumping system [1] is widely used in aerospace [2], communication [3], industrial manufacturing [4], medicine [5], power and other fields [6]. The Markov jumping system shows good adaptability in dealing with random phenomena such as packet loss, delay, channel fading and more [7] [8] [9] [10] [11]. In Ref. [12], finite-time control is designed based on $H_{\infty}$ performance for Markov jumping system with partially unknown time-varying transfer probability. A model-dependent Lyapunov function composed of bilateral closed-loop functions is constructed and applied to the fuzzy Markov jump system of sampled data in Ref. [13]. An uncertain complex network with Markov topology and random time-varying delay is studied in Ref. [14]. However, the aging [15] and damage [16] of components cannot be avoided in the actual system, the resources are wasted and the ideal control purpose is not achieved. Stability has always been one of the key points in control science. Only to ensure the stability of the system, the system can run normally. The study of the stability of the system continues. And the rapid stability of the system is also the embodiment of improving social productivity. How to make the system reach global stability faster is always one of the problems studied by many scholars. Filtering and signal technology [17] is one of the most important problems in the field of control. And the role of the filter whether on the elimination of interference signal or in the enhancement of the effective signal has a very good performance. Therefore, it is of practical significance to study the filtering problem of Markov jumping systems.

Filtering technology has always had an excellent performance in signal processing, and there are many studies on filter design, there are related designs of filtering in non-linear systems [18], time-delay systems [19], stochastic systems [20], fuzzy systems [21], etc. It is pointed out here that a time-varying filter [22] is designed to study stochastic systems, and its advantage is to obtain better transient performance. The article [23] proposes an asynchronous filter under $l_{2}-l_{\infty}$ performance, which makes full use of part of the available system modal information, improves signal quality, and saves signal resources. Next, a kind of nonstationary controller [24] is designed for a linear Markov jump system, which not only achieves the state feedback more accurately, but also shows excellent adjustment ability. If a nonstationary filter can be designed, the complex Markov jump system can be stabilized more quickly, and the signal can be used more efficiently, so it will be necessary to study the nonstationary filter.

Based on the above considerations, this paper mainly studied nonstationary state estimation for Markov jumping system with fading channel and multiplicative noises. To solve the filter gain matrix, an additional relaxation matrix consistent with the filter modes is introduced. The function of the relaxation variable is to eliminate the uncertain term in the matrix and facilitate the solution of matrix inequality by LMIs. The form of the relaxation variable is as follows:

$$
\bar{T}_{\alpha(k), l(k), \delta(k)}=\left[\begin{array}{cc}
T_{\alpha(k), l(k), \delta(k)} & U_{\alpha(k), t(k), \delta(k)} \\
O_{\alpha(k), l(k), \delta(k)} & U_{\alpha(k), l(k), \delta(k)}
\end{array}\right]<0,
$$


which are used to eliminate the non-linear terms. For Markov jumping system with random stability and $\wp$ dissipative performance index, a nonstationary state filter based on multi-segment homogeneous Markov chains dispersion is designed. Finally, the effectiveness of the method is verified by simulation. The contributions of this work are as follows:

- For Markov jumping system: The channel coefficient, which is more consistent with the actual mode, is introduced to adapt to the complex signals received by the state estimator that varies with the mode. And in actual systems, it is often unrealistic to have only one kind of noise, so multiplicative noise is considered.

- For filter: A nonstationary state estimator is designed to adjust the modal part received by the original system.

- For simulation experiment: The simulation experiments of nonstationary filter and traditional asynchronous filter are done respectively, and the results are more convincing through simulation comparison.

Notations: See Table 1.

\section{Problem Formulation}

Consider the following system:

$$
\begin{aligned}
& x(k+1)=A_{\alpha(k)} x(k)+B_{\alpha(k)} v(k)+\left[E_{\alpha(k)} x(k)+F_{\alpha(k)} v(k)\right] \xi(k), \\
& \tilde{y}(k)=C_{\alpha(k)} x(k)+G_{\alpha(k)} v(k), \\
& z(k)=L_{\alpha(k)} x(k),
\end{aligned}
$$

where $x(k) \in \mathbb{R}^{n_{x}}$ is the state vector, $\tilde{y}(k) \in \mathbb{R}^{n_{y}}$ represents the process output, $z(k) \in \mathbb{R}^{n_{z}}$ is the signal waiting to be evaluated and $v(k) \in l_{2}-l_{\infty}$ is the disturbance noise input. For each $\alpha(k)$, the system matrices $A_{\alpha(k)}, B_{\alpha(k)}$, $\alpha(k), E_{\alpha(k)}, F_{\alpha(k)}, G_{\alpha(k)}$ and $L_{\alpha(k)}$ represent the given real matrices. $\xi(k)$ represents a scalar Brownian Motion with:

$$
E\{\xi(a)\}=0, E\left\{\xi^{2}(a)\right\}=1, E\{\xi(a) \xi(b)\}=0,(a \neq b) .
$$

Table 1. Nomenclature.

\begin{tabular}{ll}
\hline $\mathbb{R} \rightarrow$ & Real number set \\
$\mathbb{R}^{n} \rightarrow$ & n-dimensional Euclidean space \\
$A^{\mathrm{T}} \rightarrow$ & The transpose of the $A$ matrix \\
$\operatorname{diag}\{\cdot\} \rightarrow$ & Block diagonal matrix \\
$E\{\cdot\} \rightarrow$ & Expectation operator relative to a probability measure \\
$\|\cdot\| \rightarrow$ & The Euclidean norm of a vector and the derived norm of a matrix \\
$* \rightarrow$ & The symmetric term in the matrix \\
$\operatorname{Pr}\{T \mid Y\} \rightarrow$ & Probability of event $T$ occurring in event $Y$ \\
$0 \rightarrow$ & A zero matrix with appropriate dimensions \\
$I_{\ell} \rightarrow$ & An identity matrix with appropriate dimensions
\end{tabular}


In system (1), the switching of system mode is up to a Markov Chain (MC) $\alpha(k) \quad\left(k \in Z^{+}\right)$. In the finite state (FS) $\mathscr{N}=\{1,2,3, \cdots, N\}$ and $\forall i, j \in \mathscr{N}$, the transition probability matrix (TPM) $\partial_{1}=\left[\aleph_{i j}\right]$ is designed,

$$
\operatorname{Pr}\left\{\alpha_{k+1}=j \mid \alpha_{k}=i\right\}=\aleph_{i j}, \quad \sum_{j=1}^{N} \aleph_{i j}=1, \quad \aleph_{i j} \in[0,1] .
$$

The measurement output $\hat{y}(k)$ is written as

$$
\hat{y}(k)=\theta_{0}(k) \tilde{y}(k)+\sum_{t=1}^{\ell} \theta_{t}(k) \tilde{y}(k-t)+H_{\alpha(k)} \omega(k),
$$

where $\omega(k) \in l_{2}-l_{\infty}$ is an external disturbance input of measurement output. $\ell=\min \{l, k\}$ with $I$ represents the given number of paths. $\theta_{t}(k)(t=0,1, \cdots, \ell)$ represents the mutually independent channel coefficients. And $\theta_{t}(k)$ are random variables and their values are in $[0,1]$ with $E\left\{\theta_{t}(k)\right\}=\bar{\theta}_{t}$ and $E\left\{\theta_{t}^{2}(k)\right\}=\mathfrak{J}_{t}^{*} . H_{\alpha(k)}$ is a given real matrices.

Remark 1 In system (1), the interference of Brownian motion $\xi(k)$, a random phenomenon, is considered. In fading channel, delay, packet loss and external disturbance $\omega(k)$ are considered. Therefore, (1)-(3) is a complex system in line with the background of industrial application.

The following asynchronous filter is designed:

$$
\begin{aligned}
& \hat{x}(k+1)=\hat{A}_{(\alpha(k), l(k), \delta(k))} \hat{x}(k)+\hat{B}_{(\alpha(k), t(k), \delta(k))} \hat{y}(k), \\
& \hat{z}(k)=\hat{L}_{(\alpha(k), t(k), \delta(k))} \hat{x}(k),
\end{aligned}
$$

where $\hat{x}(k) \in \mathbb{R}^{n_{x}}$ is the estimate of state $x(k), \hat{z}(k) \in \mathbb{R}^{n_{z}}$ is the estimate of the $z(k)$ and $\hat{A}_{(\alpha(k), l(k), \delta(k))}, \hat{B}_{(\alpha(k), t(k), \delta(k))}$ and $\hat{L}_{(\alpha(k), l(k), \delta(k))}$ are the designed gain filter matrices. In FS, $\imath(k)\left(k \in Z^{+}\right)$satisfies the discrete nonstationary MC, $/ /=\{1,2, \cdots, M\}$ and $\forall m, n \in \mathbb{l} \quad$ with TPM $\partial_{2}=\left[\pi_{m n}^{\alpha(k+1)}\right]$ given by

$$
\begin{aligned}
& \operatorname{Pr}\left\{\boldsymbol{l}_{k+1}=n \mid l_{k}=m\right\}=\pi_{m n}^{\alpha(k+1)}, \\
& \sum_{m=1}^{M} \pi_{m n}^{\alpha(k+1)}=1, \quad \pi_{m n}^{\alpha(k+1)} \in[0,1] .
\end{aligned}
$$

$\delta(k)\left(k \in Z^{+}\right)$also represents a discrete a MC in FS, $Q=\{1,2, \cdots, Q\}$ and $\forall p, q \in \mathcal{C} \quad$ with TPM $\partial_{3}=\left[\rho_{p q}^{\alpha(k+1), l(k+1)}\right]$ given by

$$
\begin{aligned}
& \operatorname{Pr}\left\{\delta_{k+1}=q \mid \delta_{k}=p\right\}=\rho_{p q}^{\alpha(k+1), l(k+1)}, \\
& \sum_{p=1}^{Q} \rho_{p q}^{\alpha(k+1), l(k+1)}=1, \quad \rho_{p q}^{\alpha(k+1), l(k+1)} \in[0,1] .
\end{aligned}
$$

Combining with system (1) and Equation (4). Letting

$$
\begin{aligned}
& \zeta(k)=\left[\begin{array}{ll}
x^{\mathrm{T}}(k) & \hat{x}^{\mathrm{T}}(k)
\end{array}\right]^{\mathrm{T}}, \bar{z}(k)=z(k)-\hat{z}(k), \\
& \tilde{\theta}_{t}(k)=\theta_{t}(k)-\bar{\theta}_{t}, \varpi(k)=\left[\begin{array}{ll}
v^{\mathrm{T}}(k) & \omega^{\mathrm{T}}(k)
\end{array}\right]^{\mathrm{T}},
\end{aligned}
$$

which needs to be noticed is $E\left\{\tilde{\theta}_{t}\right\}=0$.

Remark 2 When,$/ l=1$ and $C=1$, the filter (5) represents the homomorphic filter. When $\mathscr{C}=1, \mathscr{C}=1$ and $\mathscr{C}=1$, the filter (5) represents mode- 
independent filter. Neither the former nor the latter can effectively utilize the part of the signal received by the state estimator. In order to solve this defect, the filter (5) has three independent modes, among which not only one mode is synchronous with the original system, but also two asynchronous modes. Therefore, the filter (5) is more regulatory and can make more efficient use of the signal from the original system (1).

Then the following filtering error system (FES) is designed as:

$$
\begin{aligned}
& \zeta(k+1)=\mho_{1}(k)+\mho_{2}(k)+\mho_{3}(k), \\
& \bar{z}(k)=\bar{L}_{i m p} \zeta(k),
\end{aligned}
$$

where

$$
\begin{aligned}
& \mho_{1}(k)= \bar{A}_{i m p} \zeta(k)+\bar{B}_{i m p} \varpi(k)+\sum_{t=1}^{\ell} \bar{\theta}_{t} \bar{C}_{i m p} \zeta(k-t)+\sum_{t=1}^{\ell} \bar{\theta}_{t} \bar{G}_{i m p} \varpi(k-t), \\
& \mho_{2}(k)=\tilde{\theta}_{0}(k) \bar{C}_{i m p} \zeta(k)+\tilde{\theta}_{0}(k) \bar{G}_{i m p} \varpi(k)+\sum_{t=1}^{\ell} \tilde{\theta}_{t}(k) \bar{C}_{i m p} \zeta(k-t) \\
&+\sum_{t=1}^{\ell} \tilde{\theta}_{t}(k) \bar{G}_{i m p} \varpi(k-t), \\
& \mho_{3}(k)=D_{1}\left[E_{i} D_{1}^{\mathrm{T}} \zeta(k)+F_{i} D_{1}^{\mathrm{T}} \varpi(k)\right] \xi(k), \\
& \bar{A}_{i m p}=\left[\begin{array}{cc}
A_{i} & 0 \\
\bar{\theta}_{0} \hat{B}_{i m p} C_{i} & \hat{A}_{i m p}
\end{array}\right], \\
& \bar{B}_{i m p}=\left[\begin{array}{cc}
B_{i} & 0 \\
\bar{\theta}_{0} \hat{B}_{i m p} G_{i} & \hat{B}_{i m p} H_{i}
\end{array}\right], \\
& \bar{C}_{i m p}=\left[\begin{array}{cc}
0 & 0 \\
\hat{B}_{i m p} C_{i} & 0
\end{array}\right], \bar{G}_{i m p}=\left[\begin{array}{cc}
0 & 0 \\
\hat{B}_{i m p} G_{i} & 0
\end{array}\right], \\
& D_{1}=\left[\begin{array}{ll}
I \\
0
\end{array}\right], \bar{L}_{i m p}=\left[\begin{array}{ll}
L_{i} & -\hat{L}_{i m p}
\end{array}\right] .
\end{aligned}
$$

Then, the Definitions and Lemmas which will be used in this article are introduced:

Definition 1 [23] Under the initial modes $\alpha(0), \imath(0), \delta(0)$ and initial conditions $\zeta(0)$, when the following inequality is satisfied, FES (7) is stochastically stable.

$$
E\left\{\sum_{k=0}^{\infty}\|\zeta(k)\|^{2} \mid \zeta(0), \alpha(0), l(0), \delta(0)\right\}<\infty .
$$

Definition 2 [25] [26] Under $l_{2}-l_{\infty}$ performance index $\wp>0$, FES (7) is called stochastically stable.

$$
\sup _{k} \sqrt{\left[\|\bar{z}(k)\|^{2}\right]}<\wp \sqrt{\sum_{k=0}^{\infty}\left\|\sum_{t=0}^{\ell} \varpi(k-t)\right\|^{2}} .
$$

Lemma 1 [27] There are constant matrices $\mathfrak{R}_{11}=\mathfrak{R}_{11}^{\mathrm{T}}>0$ and $\mathfrak{R}_{22}=\mathfrak{R}_{22}^{\mathrm{T}}>0$. Then $\mathfrak{R}_{11}+\mathfrak{R}_{12} \mathfrak{R}_{22}^{-1} \mathfrak{R}_{12}^{\mathrm{T}}<0$ if and only if

$$
\left[\begin{array}{cc}
\mathfrak{R}_{11} & \mathfrak{R}_{12} \\
* & -\mathfrak{R}_{22}
\end{array}\right]<0 \quad \text { or } \quad\left[\begin{array}{cc}
-\mathfrak{R}_{22} & \mathfrak{R}_{12}^{\mathrm{T}} \\
* & \mathfrak{R}_{11}
\end{array}\right]<0
$$




\section{Main Results}

Firstly, the $l_{2} \in[0, \infty)$ performance of FES (7) is analyzed, and the following results are given.

Theorem 1 Under $l_{2}-l_{\infty}$ performance, for a given scalar $\wp>0$, then FES (7) is stochastically stable, if there exist matric $P_{i m p}>0$ and $\mathcal{P}_{i m p}>0$ such that $\forall i \in \mathscr{N}, m \in \mathscr{l}, p \in \mathcal{Q}$, Ref. (13) and Ref. (14) hold follow:

$$
\left[\begin{array}{ccccccc}
\Lambda_{i m p} & 0 & 0 & 0 & \bar{A}_{i m p}^{\mathrm{T}} \mathcal{P}_{i m p} & \sqrt{\mathfrak{I}_{0}^{*}} \bar{C}_{i m p}^{\mathrm{T}} \mathcal{P}_{i m p} & D_{1} E_{i}^{\mathrm{T}} D_{1}^{\mathrm{T}} \mathcal{P}_{i m p} \\
* & -\bar{R}_{\ell} & 0 & 0 & \bar{\Gamma}_{\ell c}^{\mathrm{T}} \mathcal{P}_{i m p} & \Gamma_{\ell c}^{* \mathrm{~T}} \mathcal{P}_{i m p} & 0 \\
* & * & -I_{\ell} & 0 & \bar{B}_{i m p}^{\mathrm{T}} \mathcal{P}_{i m p} & \sqrt{\mathfrak{J}_{0}^{*}} \bar{G}_{i m p}^{\mathrm{T}} \mathcal{P}_{i m p} & D_{1} F_{i}^{\mathrm{T}} D_{1}^{\mathrm{T}} \mathcal{P}_{i m p} \\
* & * & * & -I_{\ell} & \bar{\Gamma}_{\ell g}^{\mathrm{T}} \mathcal{P}_{i m p} & \Gamma_{\ell g}^{* \mathrm{~T}} \mathcal{P}_{i m p} & 0 \\
* & * & * & * & -\mathcal{P}_{i m p} & 0 & 0 \\
* & * & * & * & * & -\mathcal{P}_{i m p} & 0 \\
* & * & * & * & * & * & -\mathcal{P}_{i m p}
\end{array}\right]<0,
$$

where $P_{\alpha(k), l(k), \delta(k)}>0$ and $R>0$ represent the proper symmetric positive definite matrix and

$$
\begin{aligned}
& \mathcal{P}_{i m p}=\sum_{j=1}^{N} \aleph_{i j} \sum_{n=1}^{M} \pi_{m n}^{(j)} \sum_{q=1}^{Q} \rho_{p q}^{(j)(n)} P_{j n q}, \\
& \Lambda_{i m p}=-\mathcal{P}_{i m p}+R, \quad \bar{R}_{\ell}=\operatorname{diag}\left[\begin{array}{llll}
\underbrace{R}_{\ell} & R & \cdots & R
\end{array}\right], \\
& \bar{\Gamma}_{\ell c}=\operatorname{diag}\left[\bar{\theta}_{1} \bar{C}_{i m p} \quad \bar{\theta}_{2} \bar{C}_{i m p} \quad \cdots \quad \bar{\theta}_{\ell} \bar{C}_{i m p}\right], \\
& \Gamma_{\ell c}^{*}=\operatorname{diag}\left[\sqrt{\mathfrak{I}_{1}^{*}} \bar{C}_{i m p} \quad \sqrt{\mathfrak{I}_{2}^{*}} \bar{C}_{i m p} \quad \cdots \quad \sqrt{\mathfrak{I}_{\ell}^{*}} \bar{C}_{i m p}\right], \\
& \bar{\Gamma}_{\ell g}=\operatorname{diag}\left[\begin{array}{llll}
\bar{\theta}_{1} \bar{G}_{i m p} & \bar{\theta}_{2} \bar{G}_{i m p} & \cdots & \bar{\theta}_{\ell} \bar{G}_{i m p}
\end{array}\right] \text {, } \\
& \Gamma_{\ell g}^{*}=\operatorname{diag}\left[\begin{array}{llll}
\sqrt{\mathfrak{J}_{1}^{*}} \bar{G}_{i m p} & \sqrt{\mathfrak{I}_{2}^{*}} \bar{G}_{i m p} & \cdots & \sqrt{\mathfrak{I}_{\ell}^{*}} \bar{G}_{i m p}
\end{array}\right] .
\end{aligned}
$$

Proof: Consider the following Lyapunov function for system (1):

$$
\mathcal{V}(k)=\mathcal{V}_{1}(k)+\mathcal{V}_{2}(k)
$$

where

$$
\begin{aligned}
& \mathcal{V}_{1}(k)=\zeta^{\mathrm{T}}(k) P_{\alpha(k), \ell(k), \delta(k)} \zeta(k), \\
& \mathcal{V}_{2}(k)=\sum_{p=1}^{\ell} \sum_{q=k-p}^{k-1} \zeta^{\mathrm{T}}(q) R \zeta(q) .
\end{aligned}
$$

According to Equation (2), filter (5) and Equation (6) that $\alpha_{k+1}=j \in \mathscr{L}$, $\alpha_{k}=i \in \mathscr{C}, l_{k+1}=n \in \mathscr{K}, l_{k}=m \in \mathscr{K} \quad$ and $\delta_{k+1}=q \in \mathscr{C}, \delta_{k}=p \in \mathscr{C}$, the following equation is obtained,

$$
\begin{aligned}
& \operatorname{Pr}\left\{\delta_{k+1}=q, l_{k+1}=n, \alpha_{k+1}=j \mid \delta_{k}=p, l_{k}=m, \alpha_{k}=i\right\} \\
& =\operatorname{Pr}\left\{\delta_{k+1}=q \mid \delta_{k}=p, l_{k+1}=n, l_{k}=m, \alpha_{k+1}=j, \alpha_{k}=i\right\} \\
& \quad \times \operatorname{Pr}\left\{l_{k+1}=n, \alpha_{k+1}=j \mid \delta_{k}=p, l_{k}=m, \alpha_{k}=i\right\}
\end{aligned}
$$




$$
\begin{aligned}
= & \operatorname{Pr}\left\{\delta_{k+1}=q \mid \delta_{k}=p, l_{k+1}=n, l_{k}=m, \alpha_{k+1}=j, \alpha_{k}=i\right\} \\
& \times \operatorname{Pr}\left\{u_{k+1}=n \mid \delta_{k}=p, l_{k}=m, \alpha_{k+1}=j, \alpha_{k}=i\right\} \\
& \times \operatorname{Pr}\left\{\alpha_{k+1}=j \mid \delta_{k}=p, \iota_{k}=m, \alpha_{k}=i\right\} \\
= & \aleph_{i j} \pi_{m n}^{(j)} \rho_{p q}^{(j)(n)} .
\end{aligned}
$$

Along the solution of Equation (14), the following equations are satisfied:

$$
\begin{aligned}
E\left\{\Delta \mathcal{V}_{1}(k)\right\}= & E\left\{\zeta^{\mathrm{T}}(k+1) \mathcal{P}_{i m p} \zeta(k+1)-\zeta^{\mathrm{T}}(k) P_{i m p} \zeta(k)\right\} \\
= & E\left\{\mho_{1}^{\mathrm{T}}(k) \mathcal{P}_{\text {imp }} \mho_{1}(k)+\mho_{2}^{\mathrm{T}}(k) \mathcal{P}_{\text {imp }} \mho_{2}(k)\right. \\
& \left.+\mho_{3}^{\mathrm{T}}(k) \mathcal{P}_{\text {imp }} \mho_{3}(k)-\zeta^{\mathrm{T}}(k) P_{\text {imp }} \zeta(k)\right\} .
\end{aligned}
$$

and

$$
\begin{aligned}
E\left\{\Delta \mathcal{V}_{2}(k)\right\} & =E\left\{\sum_{p=1}^{\ell}\left(\sum_{q=k-p+1}^{k} \zeta^{\mathrm{T}}(q) R \zeta(q)-\sum_{q=k-p}^{k-1} \zeta^{\mathrm{T}}(q) R \zeta(q)\right)\right\} \\
& =E\left\{\sum_{p=1}^{\ell}\left(\zeta^{\mathrm{T}}(k) R \zeta(k)-\zeta^{\mathrm{T}}(k-p) R \zeta(k-p)\right)\right\} \\
& =E\left\{\zeta^{\mathrm{T}}(k) R \zeta(k)-\zeta_{\ell}^{\mathrm{T}}(k) \bar{R}_{\ell} \zeta_{\ell}(k)\right\}
\end{aligned}
$$

where

$$
\zeta_{\ell}(k)=\left[\begin{array}{llll}
\zeta^{\mathrm{T}}(k-1) & \zeta^{\mathrm{T}}(k-2) & \cdots & \zeta^{\mathrm{T}}(k-\ell)
\end{array}\right]^{\mathrm{T}} .
$$

Assuming initial conditions and nonzero $\varpi(g) \in l_{2}-l_{\infty}$, we obtain

$$
\begin{aligned}
J(k) & =\sum_{g=0}^{k-1} \mathcal{E}\left\{\Delta V_{1}(g)+\Delta V_{2}(g)-\sum_{t=0}^{\ell}\left(\varpi^{\mathrm{T}}(k-t) \varpi(k-t)\right)\right\} \\
& =\sum_{g=0}^{k-1} \mathcal{E}\left\{\chi^{\mathrm{T}}(g) \Xi_{i m p} \chi(g)\right\},
\end{aligned}
$$

where

$$
\begin{aligned}
& \chi(g)=\left[\begin{array}{llll}
\zeta^{\mathrm{T}}(g) & \zeta_{\ell}^{\mathrm{T}}(g) & \varpi^{\mathrm{T}}(g) & \varpi_{\ell}^{\mathrm{T}}(g)
\end{array}\right]^{\mathrm{T}}, \\
& \varpi_{\ell}(g)=\left[\begin{array}{llll}
\varpi^{\mathrm{T}}(g-1) & \varpi^{\mathrm{T}}(g-2) & \cdots & \varpi^{\mathrm{T}}(g-\ell)
\end{array}\right]^{\mathrm{T}}, \\
& \Xi_{i m p}=\left[\begin{array}{cccc}
\Lambda_{i m p} & 0 & 0 & 0 \\
* & -\bar{R}_{\ell} & 0 & 0 \\
* & * & -I_{\ell} & 0 \\
* & * & * & -I_{\ell}
\end{array}\right]+\left[\begin{array}{c}
\bar{A}_{i m p}^{\mathrm{T}} \\
\bar{\Gamma}_{\ell c}^{\mathrm{T}} \\
\bar{B}_{i m p}^{\mathrm{T}} \\
\bar{\Gamma}_{\ell g}^{\mathrm{T}}
\end{array}\right] \mathcal{P}_{i m p}\left[\begin{array}{c}
\bar{A}_{i m p}^{\mathrm{T}} \\
\bar{\Gamma}_{\ell c}^{\mathrm{T}} \\
\bar{B}_{i m p}^{\mathrm{T}} \\
\bar{\Gamma}_{\ell g}^{\mathrm{T}}
\end{array}\right]^{\mathrm{T}}+\left[\begin{array}{c}
\sqrt{\mathcal{\Im}_{0}^{*}} \bar{C}_{i m p}^{\mathrm{T}} \\
\Gamma_{\ell c}^{* \mathrm{~T}} \\
\sqrt{\mathcal{\Im}_{0}^{*}} \bar{G}_{i m p}^{\mathrm{T}} \\
\Gamma_{\ell g}^{* \mathrm{~T}}
\end{array}\right] \\
& \times \mathcal{P}_{i m p}\left[\begin{array}{c}
\sqrt{\widetilde{\Im}_{0}^{*}} \bar{C}_{i m p}^{\mathrm{T}} \\
\Gamma_{l c}^{* \mathrm{~T}} \\
\sqrt{\widetilde{\Im}_{0}^{*}} \bar{G}_{i m p}^{\mathrm{T}} \\
\Gamma_{l g}^{* \mathrm{~T}}
\end{array}\right]^{\mathrm{T}}+\left[\begin{array}{c}
D_{1} E_{i}^{\mathrm{T}} D_{1}^{\mathrm{T}} \\
0 \\
\bar{F}_{i m p}^{\mathrm{T}} \\
0
\end{array}\right] \mathcal{P}_{i m p}\left[\begin{array}{c}
D_{1} E_{i}^{\mathrm{T}} D_{1}^{\mathrm{T}} \\
0 \\
D_{1} \bar{F}_{i m p}^{\mathrm{T}} D_{1}^{\mathrm{T}} \\
0
\end{array}\right]^{\mathrm{T}} .
\end{aligned}
$$

Equation (11) is set up by Lemma 1.

Under initial condition, nonzero $\forall \varpi(k) \in l_{2}-l_{\infty}$ and $J(k)<0$, which is obvious that: 


$$
E\left\{\zeta^{\mathrm{T}}(k) P_{i m p} \zeta(k)\right\}=E\left\{V_{1}(k)\right\} \leq \sum_{g=0}^{k-1}\left(\sum_{t=0}^{\ell}\left(\varpi^{\mathrm{T}}(g-t) \varpi(g-t)\right)\right) .
$$

The inequality below can be obtained by Lemma 1 that Equation (12) is true

$$
\bar{L}_{i m p}^{\mathrm{T}} \bar{L}_{i m p} \leq \wp^{2} P_{i m p} \text {, }
$$

the following inequality, which implies Equation (9), is obtained

$$
\begin{aligned}
E\left\{\bar{z}^{\mathrm{T}}(k) \bar{z}(k)\right\} & =E\left\{\zeta^{\mathrm{T}}(k) \bar{L}_{\text {imp }}^{\mathrm{T}} \bar{L}_{i m p} \zeta(k)\right\} \\
& \leq \wp^{2} E\left\{\zeta(k)^{\mathrm{T}} \mathcal{P}_{\text {imp }} \zeta(k)\right\} \\
& \leq \wp^{2} E\left\{\sum_{g=0}^{k-1}\left(\sum_{t=0}^{\ell}\left(\varpi^{\mathrm{T}}(g-t) \varpi(g-t)\right)\right)\right\} \\
& \leq \wp^{2} E\left\{\sum_{g=0}^{k-1}\left(\sum_{t=0}^{\infty}\left(\varpi^{\mathrm{T}}(g-t) \varpi(g-t)\right)\right)\right\} .
\end{aligned}
$$

Next, suppose there is a scalar $\eta>0$ and consider the situation that any $\varpi(k)=0$, the following equality holds

$$
\begin{aligned}
\Xi= & {\left[\begin{array}{cc}
\Lambda_{i m p} & 0 \\
* & -\bar{R}_{\ell}
\end{array}\right]+\left[\begin{array}{c}
\bar{A}_{i m p}^{\mathrm{T}} \mathcal{P}_{i m p} \\
\bar{\Gamma}_{\ell c}^{\mathrm{T}} \mathcal{P}_{i m p}
\end{array}\right] \mathcal{P}_{i m p}\left[\begin{array}{c}
\bar{A}_{i m p}^{\mathrm{T}} \mathcal{P}_{i m p} \\
\bar{\Gamma}_{\ell c}^{\mathrm{T}} \mathcal{P}_{i m p}
\end{array}\right]^{\mathrm{T}} } \\
& +\left[\begin{array}{c}
\sqrt{\mathfrak{I}_{0}^{*}} \bar{C}_{i m p}^{\mathrm{T}} \mathcal{P}_{i m p} \\
\Gamma_{\ell c}^{* \mathrm{~T}} \mathcal{P}_{i m p}
\end{array}\right] \mathcal{P}_{i m p}\left[\begin{array}{c}
\sqrt{\mathfrak{I}_{0}^{*}} \bar{C}_{\text {imp }}^{\mathrm{T}} \mathcal{P}_{i m p} \\
\Gamma_{\ell c}^{* \mathrm{~T}} \mathcal{P}_{\text {imp }}
\end{array}\right]^{\mathrm{T}} .
\end{aligned}
$$

Then, Equation (22) is obvious that

$$
\begin{aligned}
E\{\Delta V(g)\} & =E\left\{\Delta V_{1}(g)+\Delta V_{2}(g)\right\} \\
& \leq E\left\{\left[\begin{array}{c}
\zeta(k) \\
\zeta_{\ell}(k)
\end{array}\right]^{\mathrm{T}} \Xi\left[\begin{array}{c}
\zeta(k) \\
\zeta_{\ell}(k)
\end{array}\right]\right\} \\
& \leq-\eta E\left\{\|\zeta(k)\|^{2}\right\} .
\end{aligned}
$$

By Definition 1, Equation (23) holds means that FES (7) is randomly stable.

Theorem 2: For the given $\wp>0$, if the following matrices are designed to satisfy

$$
P_{i m p}=\left[\begin{array}{cc}
P_{i m p}^{1} & P_{i m p}^{2} \\
* & P_{i m p}^{3}
\end{array}\right]>0,
$$

and matrices $T_{i m p}, O_{i m p}, U_{i m p}, A_{\text {fipm }}, B_{\text {fipm }}$ and $C_{\text {fipm }}$ such that $\forall i \in \mathscr{N}$, $\forall m \in \mathscr{l}, \forall p \in \mathscr{C}$.

$$
\begin{gathered}
{\left[\begin{array}{cc}
\Theta_{1} & \Theta_{2} \\
* & \Theta_{3}
\end{array}\right]<0,} \\
{\left[\begin{array}{ccc}
P_{i m p}^{1} & P_{i m p}^{2} & L_{i} \\
* & P_{i m p}^{3} & -\hat{L}_{i m p}^{\mathrm{T}} \\
* & * & \wp^{2} I
\end{array}\right] \geq 0,}
\end{gathered}
$$


where

$$
\begin{aligned}
& \Theta_{1}=\left[\begin{array}{cccccccc}
\Lambda_{i m p}^{1} & -\Lambda_{i m p}^{2} & 0 & 0 & 0 & 0 & 0 & 0 \\
* & -\Lambda_{i m p}^{3} & 0 & 0 & 0 & 0 & 0 & 0 \\
* & * & -\bar{R}_{\ell}^{1} & -\bar{R}_{\ell}^{2} & 0 & 0 & 0 & 0 \\
* & * & * & -\bar{R}_{\ell}^{3} & 0 & 0 & 0 & 0 \\
* & * & * & * & -I_{\ell} & 0 & 0 & 0 \\
* & * & * & * & * & -I_{\ell} & 0 & 0 \\
* & * & * & * & * & * & -I_{\ell} & 0 \\
* & * & * & * & * & * & * & -I_{\ell}
\end{array}\right],
\end{aligned}
$$

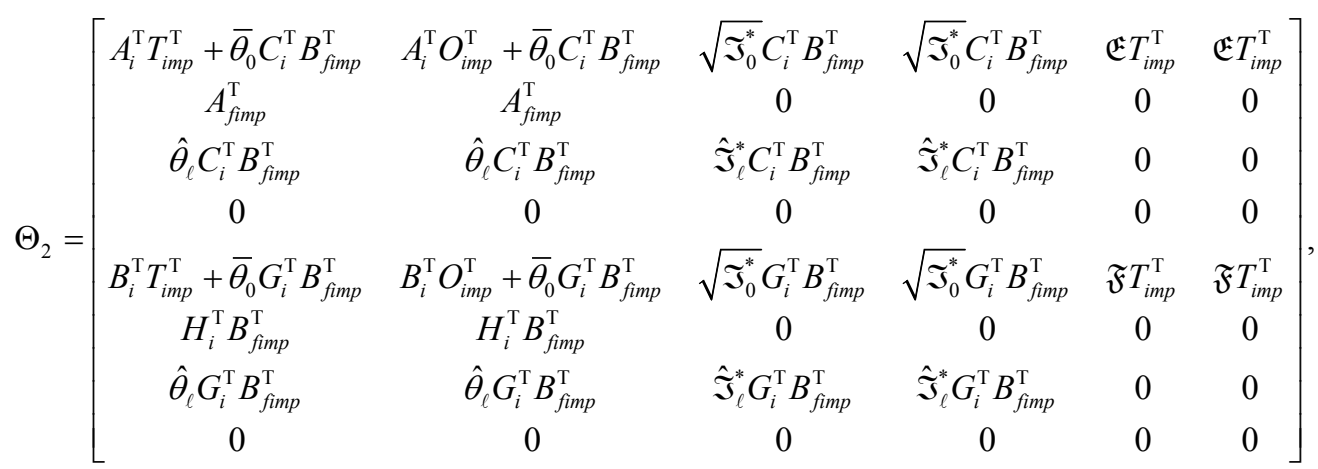

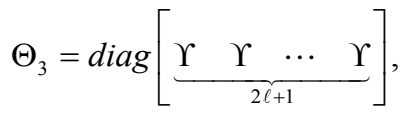

$$
\begin{aligned}
& \Lambda_{i m p}^{1}=p_{i m p}^{1}+R^{1}, \Lambda_{i m p}^{2}=p_{i m p}^{2}+R^{2}, \Lambda_{i m p}^{3}=p_{i m p}^{3}+R^{3}, \\
& \hat{\theta}_{\ell}=\operatorname{diag}\left[\bar{\theta}_{1}, \bar{\theta}_{2}, \cdots, \bar{\theta}_{\ell}\right], \quad \hat{\mathfrak{I}}_{\ell}^{*}=\operatorname{diag}\left[\mathfrak{I}_{1}^{*}, \mathfrak{I}_{2}^{*}, \cdots, \mathfrak{I}_{\ell}^{*}\right], \\
& \mathfrak{E}=D_{i} E_{i} D_{i}^{\mathrm{T}}, \quad \mathfrak{F}=D_{i} F_{i} D_{i}^{\mathrm{T}} . \\
& \Upsilon=\left[\begin{array}{cc}
\mathcal{P}_{i m p}^{1}-T_{i m p}-T_{i m p}^{\mathrm{T}} & \mathcal{P}_{i m p}^{2}-U_{i m p}-O_{i m p}^{\mathrm{T}} \\
* & \mathcal{P}_{i m p}^{3}-U_{i m p}-U_{i m p}^{\mathrm{T}}
\end{array}\right] .
\end{aligned}
$$

Proof: Choosing $\bar{T}_{i m p}=\mathcal{P}_{i m p}$, Equation (26) was immediately available from Equation (11),

$$
\left[\begin{array}{ccccccc}
\Lambda_{1} & 0 & 0 & 0 & \bar{A}_{i m p}^{\mathrm{T}} \bar{T}_{i m p}^{\mathrm{T}} & \mathfrak{I}_{0}^{*} \bar{C}_{i m p}^{\mathrm{T}} \bar{T}_{i m p}^{\mathrm{T}} & \mathfrak{E} \bar{T}_{i m p}^{\mathrm{T}} \\
* & -\bar{R}_{\ell} & 0 & 0 & \bar{\Gamma}_{\ell c}^{\mathrm{T}} \bar{T}_{i m p}^{\mathrm{T}} & \Gamma_{\ell c}^{* \mathrm{~T}} \bar{T}_{i m p}^{\mathrm{T}} & 0 \\
* & * & -I_{l} & 0 & \bar{B}_{i m p}^{\mathrm{T}} \bar{T}_{i m p}^{\mathrm{T}} & \mathfrak{I}_{0}^{*} \bar{G}_{i m p}^{\mathrm{T}} \bar{T}_{i m p}^{\mathrm{T}} & \mathfrak{F} \bar{T}_{i m p}^{\mathrm{T}} \\
* & * & * & -I_{l} & \bar{\Gamma}_{\ell g}^{\mathrm{T}} \bar{T}_{i m p}^{\mathrm{T}} & \Gamma_{\ell g}^{* \mathrm{~T}} \bar{T}_{i m p}^{\mathrm{T}} & 0 \\
* & * & * & * & \mathcal{P}_{i m p}-\bar{T}_{i m p}^{\mathrm{T}}-\bar{T}_{i m p}^{\mathrm{T}} & 0 & 0 \\
* & * & * & * & * & \mathcal{P}_{i m p}-\bar{T}_{i m p}-\bar{T}_{i m p}^{\mathrm{T}} & 0 \\
* & * & * & * & * & * & \mathcal{P}_{i m p}-\bar{T}_{i m p}-\bar{T}_{i m p}^{\mathrm{T}}
\end{array}\right]<0 .
$$

Let's take Equation (26) left times Equation (27) and right times Equation (27) transpose

$$
\operatorname{diag}\left[\begin{array}{llllllll}
I & I & I & I & I & \bar{T}_{i m p} \mathcal{P}_{i m p}^{-1} & \bar{T}_{i m p} \mathcal{P}_{i m p}^{-1}
\end{array}\right]
$$

Equation (28) is obviously taken from Equation (26) and Equation (27) that 


$$
\begin{aligned}
& {\left[\begin{array}{ccccccc}
\Lambda_{1} & 0 & 0 & 0 & \bar{A}_{i m p}^{\mathrm{T}} \bar{T}_{i m p}^{\mathrm{T}} & \mathfrak{\Im}_{0}^{*} \bar{C}_{i m p}^{\mathrm{T}} \bar{T}_{i m p}^{\mathrm{T}} & \mathfrak{E} \bar{T}_{i m p}^{\mathrm{T}} \\
* & -\bar{R}_{\ell} & 0 & 0 & \bar{\Gamma}_{l c}^{\mathrm{T}} \bar{T}_{i m p}^{\mathrm{T}} & \Gamma_{l c}^{\mathrm{T}} \bar{T}_{i m p}^{\mathrm{T}} & 0 \\
* & * & -I_{l} & 0 & \bar{B}_{i m p}^{\mathrm{T}} \bar{T}_{i m p}^{\mathrm{T}} & \mathfrak{\Im}_{0}^{*} \bar{G}_{i m p}^{\mathrm{T}} \bar{T}_{i m p}^{\mathrm{T}} & \mathfrak{F} \bar{T}_{i m p}^{\mathrm{T}} \\
* & * & * & -I_{l} & \bar{\Gamma}_{\ell g}^{\mathrm{T}} \bar{T}_{i m p}^{\mathrm{T}} & \Gamma_{\ell g}^{\mathrm{T}} \bar{T}_{i m p}^{\mathrm{T}} & 0 \\
* & * & * & * & \overline{\mathcal{Y}} & 0 & 0 \\
* & * & * & * & * & \overline{\mathcal{Y}} & 0 \\
* & * & * & * & * & * & \overline{\mathcal{Y}}
\end{array}\right]<0,} \\
& {\left[\begin{array}{ccc}
P_{i m p}^{1} & P_{i m p}^{2} & L_{i} \\
* & P_{i m p}^{3} & -\hat{L}_{i m p} \\
* & * & \wp^{2} I
\end{array}\right] \geq 0,}
\end{aligned}
$$

where $\overline{\mathcal{Y}}=-\bar{T}_{\text {imp }} \mathcal{P}_{\text {imp }} \bar{T}_{\text {imp }}^{\mathrm{T}}$.

In addition, since $\left(\mathcal{P}_{\text {imp }}-\bar{T}_{\text {imp }}\right) \mathcal{P}_{\text {imp }}^{-1}\left(\mathcal{P}_{\text {imp }}-\bar{T}_{\text {imp }}\right)^{\mathrm{T}} \geq 0$, we have:

$$
-\bar{T}_{i m p} \mathcal{P}_{i m p}^{-1} \bar{T}_{i m p}^{\mathrm{T}} \leq \mathcal{P}_{\text {imp }}-\bar{T}_{i m p}-\bar{T}_{i m p}^{\mathrm{T}} \text {. }
$$

To sum up, if Equation (28) is true then Equation (11) is clearly true, let's introduce the relaxation variable to eliminate the products between System-matrix and Lyapunov-matrix. The substitution in Equation (28) is represented like this

$$
\begin{aligned}
& P_{i m p}=\left[\begin{array}{cc}
p_{i m p}^{1} & p_{i m p}^{2} \\
* & p_{i m p}^{3}
\end{array}\right], R=\left[\begin{array}{cc}
R^{1} & R^{2} \\
* & R^{3}
\end{array}\right], \bar{T}_{i m p}=\left[\begin{array}{ll}
T_{i m p} & U_{i m p} \\
O_{i m p} & U_{i m p}
\end{array}\right], \\
& A_{f i m p}=U_{i m p} \hat{A}_{i m p}, \quad B_{f i m p}=U_{i m p} \hat{B}_{i m p}, \quad L_{f i m p}=\bar{L}_{i m p} .
\end{aligned}
$$

Then Equation (24) is got from the above deduction. And then, by $P_{i m p}=\left[\begin{array}{cc}P_{i m p}^{1} & P_{i m p}^{2} \\ * & P_{i m p}^{3}\end{array}\right]$ and $\bar{L}_{i m p}=\left[\begin{array}{ll}L_{i} & -\hat{L}_{i m p}\end{array}\right]$, we can just go straight from Equation (12) to Equation (25).

\section{A Numerical Example}

As set of examples are taken to verify the positive value of the method. $x(0)=\left[\begin{array}{ll}0.17 & 0.17\end{array}\right]^{\mathrm{T}}$ is selected as the initial state. Applying the disturbance noise $v(k)=1 / k^{2}+1$ in system (1) and the external disturbance $\omega(k)=1 /(k+1)^{2}$ in Equation (3). The parameters are given by

$$
\begin{gathered}
A_{1}=\left[\begin{array}{ll}
0.74 & 0.241 \\
0.86 & -0.62
\end{array}\right], A_{2}=\left[\begin{array}{cc}
-0.1 & 0.4 \\
-0.5 & 1
\end{array}\right], \\
B_{1}=\left[\begin{array}{l}
1.6 \\
0.5
\end{array}\right], B_{2}=\left[\begin{array}{l}
0.3 \\
0.2
\end{array}\right], C_{1}=\left[\begin{array}{ll}
-0.1 & 1
\end{array}\right], \\
C_{2}=\left[\begin{array}{ll}
-0.1 & 1
\end{array}\right], E_{1}=\left[\begin{array}{cc}
-0.1 & 0.2 \\
-0.3 & -0.4
\end{array}\right], \\
E_{2}=\left[\begin{array}{cc}
-0.2 & 0.1 \\
0.4 & -0.5
\end{array}\right], F_{1}=\left[\begin{array}{ll}
0.5 & 1
\end{array}\right], \\
F_{2}=\left[\begin{array}{ll}
1 & 0
\end{array}\right], L_{1}=\left[\begin{array}{ll}
1 & 0
\end{array}\right], L_{2}=\left[\begin{array}{ll}
0.1 & 0
\end{array}\right], \\
H_{1}=0.2, H_{2}=0.3, G_{1}=0.3, G_{2}=0.4 .
\end{gathered}
$$


The TPM of MC $\alpha(k)$ is taken as

$$
\partial_{1}=\left[\begin{array}{ll}
0.3 & 0.7 \\
0.4 & 0.6
\end{array}\right]
$$

the TPMs of MC $\imath(k)$ are given by

$$
\partial_{2}^{1}=\left[\begin{array}{ll}
0.1 & 0.9 \\
0.7 & 0.3
\end{array}\right], \partial_{2}^{2}=\left[\begin{array}{ll}
0.3 & 0.7 \\
0.4 & 0.6
\end{array}\right],
$$

and the TPMs of MC $\delta(k)$ are chosen as

$$
\begin{aligned}
& \partial_{3}^{1,1}=\left[\begin{array}{ll}
0.3 & 0.7 \\
0.7 & 0.3
\end{array}\right], \partial_{3}^{1,2}=\left[\begin{array}{ll}
0.43 & 0.57 \\
0.55 & 0.45
\end{array}\right], \\
& \partial_{3}^{2,1}=\left[\begin{array}{ll}
0.77 & 0.23 \\
0.42 & 0.58
\end{array}\right], \partial_{3}^{2,2}=\left[\begin{array}{cc}
0.55 & 0.45 \\
0.3 & 0.7
\end{array}\right] .
\end{aligned}
$$

The order of the fading model is $l=1$ and the probability density functions $\varepsilon(\cdot)$ of channel coefficients are represented as follows

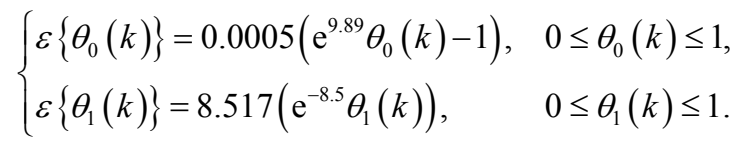

The mathematical expectation $\bar{\theta}_{0}=0.8991, \bar{\theta}_{1}=0.1174$ and variance $\mathfrak{I}_{0}^{*}=0.0133, \mathfrak{I}_{1}^{*}=0.01364$. By solving the LMIs of Theorem 2, the parameters of the nonstationary filter are obtained as

$$
\begin{aligned}
& \hat{A}_{111}=\left[\begin{array}{cc}
-0.0168 & 0.2756 \\
0.0916 & 0.0546
\end{array}\right], \quad \hat{B}_{111}=\left[\begin{array}{c}
-0.8720 \\
-0.5687
\end{array}\right], \quad \hat{L}_{111}=\left[\begin{array}{ll}
-0.3748 & -0.0224
\end{array}\right], \\
& \hat{A}_{112}=\left[\begin{array}{ll}
-0.0310 & 0.0099 \\
-0.0392 & 0.1800
\end{array}\right], \quad \hat{B}_{112}=\left[\begin{array}{l}
-0.8208 \\
-0.2109
\end{array}\right], \quad \hat{L}_{112}=\left[\begin{array}{ll}
-0.4419 & -0.1792
\end{array}\right] \text {, } \\
& \hat{A}_{121}=\left[\begin{array}{cc}
0.0501 & 0.2471 \\
0.1036 & -0.0907
\end{array}\right], \quad \hat{B}_{121}=\left[\begin{array}{c}
-0.9696 \\
-0.5837
\end{array}\right], \quad \hat{L}_{121}=\left[\begin{array}{ll}
-0.3745 & 0.0327
\end{array}\right] \text {, } \\
& \hat{A}_{122}=\left[\begin{array}{cc}
0.0399 & -0.1827 \\
-0.1340 & -0.0350
\end{array}\right], \quad \hat{B}_{122}=\left[\begin{array}{c}
-0.1029 \\
-0.0348
\end{array}\right], \quad \hat{L}_{122}=\left[\begin{array}{ll}
0.0169 & -0.0638
\end{array}\right] \text {, } \\
& \hat{A}_{211}=\left[\begin{array}{cc}
-0.2022 & -0.0681 \\
0.1506 & 0.2166
\end{array}\right], \quad \hat{B}_{211}=\left[\begin{array}{c}
-0.1215 \\
-0.3408
\end{array}\right], \quad \hat{L}_{211}=\left[\begin{array}{ll}
-0.1095 & -0.0518
\end{array}\right] \text {, } \\
& \hat{A}_{212}=\left[\begin{array}{cc}
0.1217 & 0.0829 \\
0.0750 & -0.0455
\end{array}\right], \quad \hat{B}_{212}=\left[\begin{array}{c}
-0.3108 \\
-0.3899
\end{array}\right], \quad \hat{L}_{212}=\left[\begin{array}{ll}
-0.0267 & 0.0524
\end{array}\right] \text {, } \\
& \hat{A}_{221}=\left[\begin{array}{cc}
-0.0704 & -0.1985 \\
-0.1167 & 0.0415
\end{array}\right], \quad \hat{B}_{221}=\left[\begin{array}{c}
-0.0727 \\
-0.0989
\end{array}\right], \quad \hat{L}_{221}=\left[\begin{array}{ll}
-0.0223 & -0.0959
\end{array}\right] \text {, } \\
& \hat{A}_{222}=\left[\begin{array}{ll}
0.1507 & -0.0706 \\
0.0129 & -0.1912
\end{array}\right], \quad \hat{B}_{222}=\left[\begin{array}{l}
-0.7374 \\
-0.2063
\end{array}\right], \quad \hat{L}_{222}=\left[\begin{array}{ll}
-0.2370 & -0.1077
\end{array}\right] \text {. }
\end{aligned}
$$

When $\mathscr{T}=1, \mathscr{C}=1$ and $C=1$, the filter in (4) is mode-independent (MIF). When . $/ /=1$ and $C=1$, the filter in (4) is called mode-dependent filter (MDF). The traditional asynchronous filter occurs when $/ /=1$ or $Q=1$. 
The following Table 2 shows the $\wp$ values of different $\bar{\theta}_{t}$ for the nonstationary and asynchronous filters under the $l_{2}-l_{\infty}$ performance index. From Table 2, that the nonstationary filter has less conservatism than asynchronous filter. The nonstationary filter can make the system (1) achieve better performance.

The relationship between the jumps of the three modes and the time series is described in Figure 1. Figure 2 shows the process of the regional stability of the system state $x(k)$ in the time series under the adjustment of the nonstationary filter and the asynchronous filter. Figure 3 and Figure 4 respectively show the stabilization process of the state $\hat{x}(k)$ of the two filters themselves and the stabilization process of the corresponding filter error system state $\zeta(k)$. Figure 5 respectively shows the process of the nonstationary filter and asynchronous filter in estimating the stability of the original system signal $\hat{z}(k)$. In terms of system state $x(k)$, filter state $\hat{x}(k)$ and filter error system state $\zeta(k)$, the nonstationary filter converge to stable faster than traditional asynchronous filter. The nonstationary filter also performs better in the estimation signal $\hat{z}(k)$. What needs to be pointed out here is that in Figure 4, although the nonstationary filter error system state produces a longer wavelength before reaching stability, it still reaches stability relatively quickly.
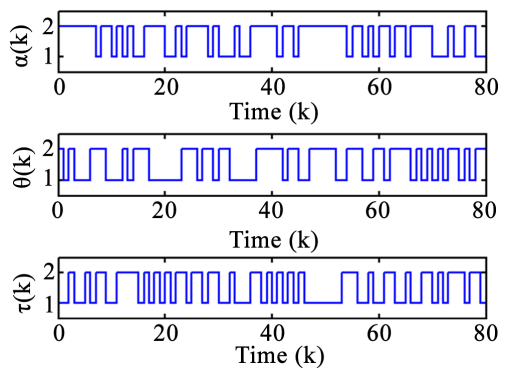

Figure 1. Three Markov chains $\alpha(k), \imath(k), \delta(k)$.

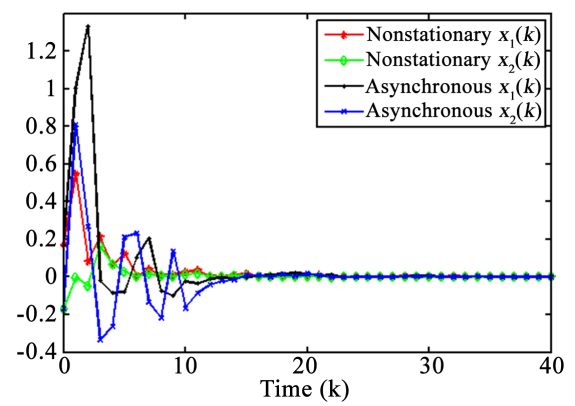

Figure 2. The state of system $x(k)$.

Table 2. Minimum $\wp$ for different kinds of filters.

\begin{tabular}{|c|c|c|c|c|c|}
\hline $\bar{\theta}_{t}$ & 0.1 & 0.3 & 0.5 & 0.7 & 0.9 \\
\hline Nonstationary filter $\wp$ & 1.2468 & 1.3219 & 1.3303 & 1.3749 & 1.3520 \\
\hline Asynchronous filter $\wp$ & 1.5168 & 1.4904 & 1.4357 & 1.4535 & 1.5364 \\
\hline
\end{tabular}




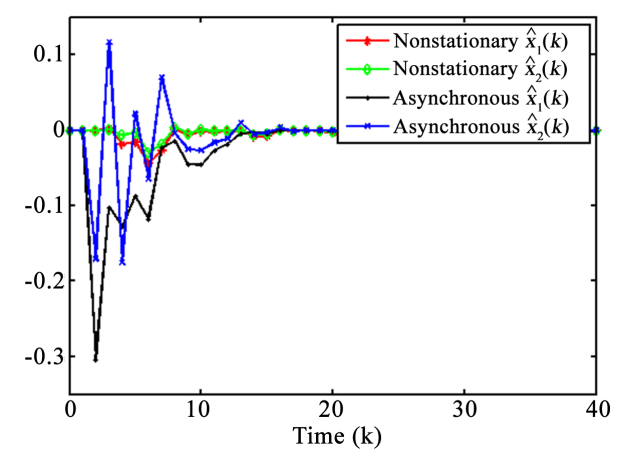

Figure 3. The state of filter $\hat{x}(k)$.

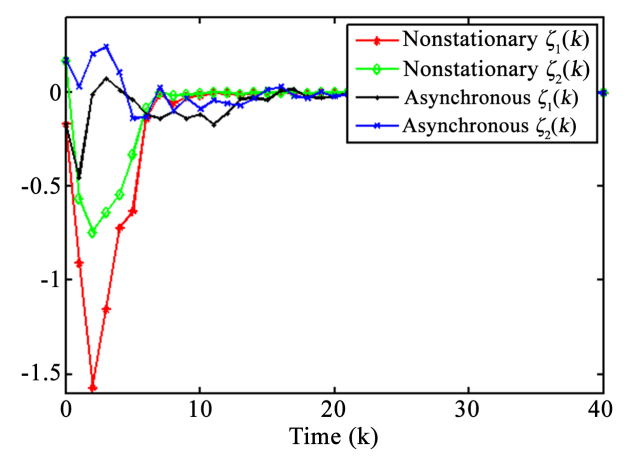

Figure 4. The state of FES $\zeta(k)$.

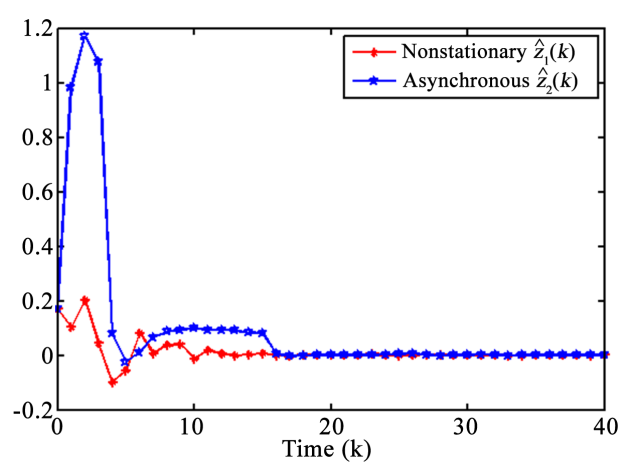

Figure 5. The estimated signal of $\bar{z}(k)$.

To sum up, the proposed nonstationary filter has better adaptability and stronger regulation, so that the system can operate effectively in the conditional industrial environment. On the other hand, compared with the traditional asynchronous filter, the nonstationary filter is more efficient to make the system stable, and has more practical engineering application significance, and is more suitable for practical engineering application promotion.

\section{Conclusion}

In this paper, the nonstationary filtering of Markov jumping system with fading channel and multiplicative noises are studied. The nonstationary filter takes into account three different segments of Markov chain, so that the nonstationary fil- 
ter not only has the same mode as the original system (1), but also provides the adjustment function for other changes in the system (1) during the mode conversion, and makes more efficient use of the signals available in the system. The designed filtering method ensures that the FES (7) is stochastically stable under the $l_{2}-l_{\infty}$ performance index. Finally, the effectiveness of the method is demonstrated based on numerical examples. It needs to be pointed out that the actual control environment is far more demanding, and the nonstationary filter can be further studied through the following aspects. For example, To avoid the defect caused by slow algorithm operation when dealing with large-scale control system, the fuzzy control in [28] can be combined to simplify the solution of filtering scheme; The performance of nonstationary filter with more time delay should be analyzed in combination with the mean residence time [29]; the nonstationary filter can be combined with the practical application of non-convex regularized image denoising [30] for more complex image processing research. And a series of works are waiting for further research.

\section{Conflicts of Interest}

The authors declare no conflicts of interest regarding the publication of this paper.

\section{References}

[1] Krasovskiü, N.N. and Lidskiü, È.A. (1961) Analytical Design of Controllers in Systems with Random Attributes. I. Statement of the Problem, Method of Solving. AVtomat: $\mathrm{i}$ Telemeh, 22, 1145-1150.

[2] Newbold, P.M. and Ho, Y.C. (2010) Detection of Changes in the Characteristics of a Gauss-Markov Process. IEEE Transactions on Aerospace and Electronic Systems, AES-4, 707-718. https://doi.org/10.1109/TAES.1968.5408688

[3] Wang, D., Ren, P. and Cheng, J. (2018) Cooperative Secure Communication in Two-Hop Buffer-Aided Networks. IEEE Transactions on Communications, 66, 972-985. https://doi.org/10.1109/TCOMM.2017.2776114

[4] Alina, R., Nikhil, S., Alexander, P., Rickert, M. and Knoll, A. (2015) Multimodal Human Activity Recognition for Industrial Manufacturing Processes in Robotic Workcells. Proceedings of the 2015 ACM on International Conference on Multimodal Interaction, Seattle, Washington DC, November 2015, 259-266. https://doi.org/10.1145/2818346.2820738

[5] Beck, J.R. and Pauker, S.G. (1983) The Markov Process in Medical Prognosis. Medical Decision Making, 3, 419-458. https://doi.org/10.1177/0272989X8300300403

[6] Hong, Y.Y. and Lian, R.C. (2012) Optimal Sizing of Hybrid Wind/PV/Diesel Generation in a Stand-Alone Power System Using Markov-Based Genetic Algorithm. IEEE Transactions on Power Delivery, 27, 640-647. https://doi.org/10.1109/TPWRD.2011.2177102

[7] Cheng, J., Park, J.H., Liu, Y., Liu, Z. and Tang, L. (2016) Finite-Time [Formula Omitted] Fuzzy Control of Nonlinear Markovian Jump Delayed Systems with Partly Uncertain Transition Descriptions. Fuzzy Sets and Systems, 314, 99-115. https://doi.org/10.1016/j.fss.2016.06.007

[8] Song, J., Niu, Y. and Zou, Y. (2018) Asynchronous Sliding Mode Control of Marko- 
vian Jump Systems with Time-Varying Delays and Partly Accessible Mode Detection Probabilities. Automatica, 93, 33-41.

https://doi.org/10.1016/j.automatica.2018.03.037

[9] Tao, J., Lu, R., Shi, P., Su, H. and Wu, Z.G. (2016) Dissipativity-Based Reliable Control for Fuzzy Markov Jump Systems with Actuator Faults. IEEE Transactions on Cybernetics, 47, 2377-2388. https://doi.org/10.1109/TCYB.2016.2584087

[10] Wei, B., Xiao, F. and Shi, Y. (2020) Synchronization in Kuramoto Oscillator Networks with Sampled-Data Updating Law. IEEE Transactions on Cybernetics, 50, 2380-2388. https://doi.org/10.1109/TCYB.2019.2940987

[11] Xu, Y., Lu, R., Peng, H., Xie, K. and Xue, A. (2015) Asynchronous Dissipative State Estimation for Stochastic Complex Networks with Quantized Jumping Coupling and Uncertain Measurements. IEEE Transactions on Neural Networks and Learning Systems, 28, 268-277. https://doi.org/10.1109/TNNLS.2015.2503772

[12] Bolzern, P., Colaneri, P. and De Nicolao, G. (2014) Stochastic Stability of Positive Markov Jump Linear Systems. Automatica, 50, 1181-1187.

https://doi.org/10.1016/j.automatica.2014.02.016

[13] Cheng, J., Zhu, H., Zhong, S., Zeng, Y. and Hou, L. (2014) Finite-Time $H_{\infty}$ Filtering for a Class of Discrete-Time Markovian Jump Systems with Partly Unknown Transition Probabilities. International Journal of Adaptive Control \& Signal Processing, 28, 1024-1042. https://doi.org/10.1002/acs.2425

[14] Tan, X., Cao, J. and Rutkowski, L. (2019) Distributed Dynamic Self-Triggered Control for Uncertain Complex Networks with Markov Switching Topologies and Random Time-Varying Delay. IEEE Transactions on Network Science and Engineering, 7, 1111-1120. https://doi.org/10.1109/TNSE.2019.2905758

[15] Matolia, S., Guduru, G., Gottumukkala, B. and Kuna, K.K. (2020) An Investigation into the Influence of Aging and Rejuvenation on Surface Free Energy Components and Chemical Composition of Bitumen. Construction and Building Materials, 245, Article ID: 118378. https://doi.org/10.1016/j.conbuildmat.2020.118378

[16] Wei, J., Wang, H., Lin, B., Sui, T., Zhao, F. and Fang, S. (2019) Acoustic Emission Signal of Fiber-Reinforced Composite Grinding: Frequency Components and Damage Pattern Recognition. International Journal of Advanced Manufacturing Technology, 103, 1403-1404. https://doi.org/10.1007/s00170-019-03782-3

[17] Nagpal, K.M. and Khargonekar, P.P. (2002) Filtering and Smoothing in an $H_{\text {finity }}$ Setting. IEEE Transactions on Automatic Control, 36, 152-166. https://doi.org/10.1109/9.67291

[18] Shen, B., Wang, Z., Ding, D. and Shu, H. (2013) $H_{\infty}$ State Estimation for Complex Networks with Uncertain Inner Coupling and Incomplete Measurements. IEEE Transactions on Neural Networks and Learning Systems, 24, 2027-2037. https://doi.org/10.1109/TNNLS.2013.2271357

[19] Zhang, B. and Zhuang, G. (2019) Extended Dissipative Control and Filtering for Singular Time-Delay Systems with Markovian Jumping Parameters. In: Gao, Q. and Karimi, H.R., Eds., Stability, Control and Application of Time-Delay Systems, Butterworth-Heinemann, Oxford, 227-255. https://doi.org/10.1016/B978-0-12-814928-7.00011-1

[20] Dragan, V, Aberkane, S. and Popa, I.L. (2018) Optimal Filtering for a Class of Linear Itô Stochastic Systems: The Dichotomic Case. Automatica, 90, 47-53. https://doi.org/10.1016/j.automatica.2017.12.025

[21] Chibani, A., Chadli, M., Ding, S.X. and Braiek, N.B. (2018) Design of Robust Fuzzy Fault Detection Filter for Polynomial Fuzzy Systems with New Finite Frequency 
Specifications. Automatica, 93, 42-54.

https://doi.org/10.1016/j.automatica.2018.03.024

[22] Dong, H., Wang, Z., Ding, S.X. and Gao, H. (2015) Event-Based $H_{\infty}$ Filter Design for a Class of Nonlinear Time-Varying Systems with Fading Channels and Multiplicative Noises. IEEE Transactions on Signal Processing, 63, 3387-3395. https://doi.org/10.1109/TSP.2015.2422676

[23] Wu, Z.G., Shi, P., Su, H. and Chu, J. (2014) Asynchronous $l_{2}-l_{\infty}$ Filtering for Discrete-Time Stochastic Markov Jump Systems with Randomly Occurred Sensor Nonlinearities. Automatica, 50, 180-186. https://doi.org/10.1016/j.automatica.2013.09.041

[24] Zhu, Y., Zhang, L. and Basin, M.V. (2016) Nonstationary $H_{\infty}$ Dynamic Output Feedback Control for Discrete Time Markov Jump Linear Systems with Actuator and Sensor Saturations. International Journal of Robust and Nonlinear Control, 26, 1010-1025. https://doi.org/10.1002/rnc.3348

[25] He, S. and Liu, F. (2012) Finite-Time $H_{\infty}$ Fuzzy Control of Nonlinear Jump System with Time Delays via Dynamic Observer-Based State Feedback. IEEE Transactions on Fuzzy Systems, 20, 605-614. https://doi.org/10.1109/TFUZZ.2011.2177842

[26] Shen, M., Yan, S., Zhang, G. and Park, J. (2016) Finite-Time $H_{\infty}$ Static Output Control of Markov Jump Systems with an Auxiliary Approach. Applied Mathematics \& Computation, 273, 553-561. https://doi.org/10.1016/j.amc.2015.10.038

[27] Shen, H., Jiao, S., Huo, S., Chen, M., Li, J. and Chen, B. (2017) On Energy-to-Peak Filtering for Semi-Markov Jump Singular Systems with Unideal Measurements. Signal Processing, 144, 127-133. https://doi.org/10.1016/j.sigpro.2017.10.013

[28] He, S. and Liu, F. (2009) Fuzzy Model-Based Fault Detection for Markov Jump Systems. International Journal of Robust and Nonlinear Control, 19, 1248-1266. https://doi.org/10.1002/rnc.1380

[29] Wu, Z.G., Shi, P., Su, H. and Chu, J. (2013) Stochastic Synchronization of Markovian Jump Neural Networks with Time-Varying Delay Using Sampled Data. IEEE Transactions on Cybernetics, 43, 1796-1806. https://doi.org/10.1109/TSMCB.2012.2230441

[30] Tang, L., Ren, Y., Fang, Z. and He, C. (2019) A Generalized Hybrid Nonconvex Variational Regularization Model for Staircase Reduction in Image Restoration. Neurocomputing, 359, 15-31. https://doi.org/10.1016/j.neucom.2019.05.073 